\title{
3 Terminitor: Cleavage Site Prediction Using Deep Learning Models
}

5 Chen Yang ${ }^{1,2}$, Chenkai Li ${ }^{1,2}$, Ka Ming Nip ${ }^{1,2}$, René L Warren ${ }^{1}$, Inanc Birol ${ }^{1,2^{*}}$

6

$7 \quad{ }^{1}$ Canada's Michael Smith Genome Sciences Centre, BC Cancer, Vancouver, V5Z 4S6, Canada

$8{ }^{2}$ University of British Columbia, Vancouver, V6T 1Z4, Canada

9

$10 *$ Corresponding author

11 E-mail: ibirol@bcgsc.ca (Birol I) 


\section{Abstract}

14 As a widespread RNA processing machinery, alternative polyadenylation plays a crucial role in

15 gene regulation. To help decipher its underlying mechanism and understand its impact, it is

16 desirable to comprehensively profile 3 '-untranslated region cleavage and associated

17 polyadenylation sites. State-of-the-art polyadenylation site detection tools are known to be

18 influenced by library preparation artefacts or manually selected features. Moreover, recently

19 published machine learning methods have only been tested on pre-constructed datasets, thus

20 lacking validation on experimental data. Here we present Terminitor, the first deep neural

21 network-based profiling pipeline to make predictions from RNA-seq data. We show how

22 Terminitor outperforms competing tools in sensitivity and precision on experimental

23 transcriptome sequencing data, and demonstrate its use with data from short- and long-read

24 sequencing technologies. For species without a good reference transcriptome annotation,

25 Terminitor is still able to pass on the information learnt from a related species and make

26 reasonable predictions. We used Terminitor to showcase how single nucleotide variations can

27 create or destroy polyadenylated cleavage sites in human RNA-seq samples. 


\section{Author Summary}

303 ' cleavage and polyadenylation of pre-mRNA is part of RNA maturation process. One gene can

31 be cleaved at different positions at its $3^{\prime}$ end, namely alternatively polyadenylation, thus

32 identifying the correct polyadenylated cleavage site (poly $(A) C S$ ) is essential to unveil its role in

33 gene regulation under different physiological and pathological conditions. The current poly(A) CS

34 prediction tools are either heavily influenced by RNA-Seq library preparation artefacts or have

35 only been designed and tested on ad hoc datasets, lacking association with real world

36 applications. In this study, we present a deep learning model, Terminitor, that predicts the

37 probability of a nucleotide sequence containing a poly $(A) C S$, and validated its performance on

38 human and mouse data. Along with the model, we propose a poly(A) CS profiling pipeline for

39 RNA-seq data. We benchmarked our pipeline against competing tools and achieved higher

40 sensitivity and precision in experimental data. The usage of Terminitor is not limited to genome

41 and transcriptome annotation and we expect it to facilitate the identification of novel isoforms,

42 improve the accuracy of transcript quantification and differential expression analysis, and

43 contribute to the repertoire of reference transcriptome annotation. 


\section{Introduction}

45 Since polyadenylated (poly $(A))$ sequences were first discovered on the $3^{\prime}$ ends of eukaryotic

46 mRNAs 40 years ago [1], numerous studies have contributed to the understanding of the

47 mechanisms, evolution, regulation, and impacts of polyadenylation [2-5]. As an essential step in

48 mRNA maturation, RNA polyadenylation involves two phases: 3' end cleavage of nascent RNA

49 and addition of a poly $(\mathrm{A})$ tail. This process is facilitated by several cis-acting RNA elements,

50 represented by the poly $(A)$ signal (PAS), conserved hexamer motifs with AATAAA being the most

51 strongest, located 10-30 nt upstream of the cleavage site (CS) [6]. Because most nascent RNA in

52 eukaryotes have more than one possible 3' end CS, mRNAs with different 3' ends are formed

53 following these two coupled processes [7]. This phenomenon is also known as alternative

54 polyadenylation (APA), which results in transcripts with different $3^{\prime}$ untranslated regions (UTR).

55 In recent years, advances in DNA/RNA sequencing technology allowed detailed study of APA

56 modulation under different physiological and pathological conditions, and shed light on its

57 implications in some diseases, especially cancer [5,8-10].

58 The importance of profiling 3' UTR cleavage

59 APA, together with alternative splicing, has considerable impact on the modulation of gene

60 expression and contributes to transcriptome complexity. As of date, the catalogue of profiled

61 poly (A) CS, especially cancer-specific ones, is far from complete [11]. Ensembl annotation version

62 GRCh38.94 records 63,620 poly(A) CS for 19,907 protein coding genes in human, and among

63 them, 73.13\% genes can produce two or more APA isoforms (S1 Fig) [12]. The most recent poly(A)

64 database, PolyA_DB3, compiled 24 human samples and cataloged 108,042 poly(A) CS for 20,998

65 genes [13]. The substantial improvement in 3' end annotation compared to Ensembl suggests the 
sample-specific usage of poly(A) CS, highlighting the incompleteness of the current annotation.

67 Moreover, usage of these poly(A) CS is dynamically regulated under different developmental stages and pathological changes, as elucidated in previous studies [5,8,14,15]. Profiling poly(A)

69 CS with respect to biological conditions serves as the first step towards deciphering the underlying mechanism of APA-mediated gene regulation. Hence, it is desirable to incorporate a

71 fast and robust poly $(\mathrm{A})$ CS characterization tool into standard transcriptome analysis pipelines.

\section{Current methods and their limitations}

In the past decades, continuous efforts have been made to annotate $3^{\prime}$ ends in the genome

74 and predict poly(A) CS in the transcriptome, using methods designed for two major sequencing

75 protocols: 3' end targeted sequencing and poly(A)-selected RNA-seq. The former is a specialized

76 high throughput sequencing technique that is developed to overcome the low sequence

77 complexity at the $3^{\prime}$ end of transcripts. These methods, including TAIL-Seq, PolyA-Seq, PAS-seq,

78 and PAT-seq [16-19], only interrogate the 3 ' ends of transcripts by primers, followed by

79 sequencing and bespoke analysis pipelines. Compared to methods that use RNA-seq, a critical

80 advantage of these protocols is their high sensitivity in detecting poly(A) CS from lowly expressed

81 transcripts. With the help of $3^{\prime}$ end sequencing technologies, several poly(A) CS databases have

82 been established [13,20-22]. Though powerful, they require specialized library preparation,

83 which can be costly and laborious, and their utility is restricted to poly $(A)$ detection. As a result,

84 they have not yet been widely adopted in genetic research.

85 In contrast, RNA-seq is the established sequencing method of choice for a wide range of 86 transcriptomics projects. It can also be used for poly(A) CS profiling on a per-sample basis, as well 87 as for systematic APA analysis of large cohorts of individuals, including retrospective studies. 
88 Poly(A) CS prediction and annotation tools designed for RNA-seq have demonstrated that with

89 suitably redundant coverage (read coverage $\geq 2$ ), RNA-seq data type contains sufficient

90 information for the identification of poly(A) tails, and thus permits the discovery of novel poly(A)

91 CS [23,24]. Generally speaking, these tools can be classified into three approaches: read-evidence

92 based, expression level based, and machine learning based.

93 Read-evidence based approaches, such as KLEAT [23] and ContextMap2 [24], search for

94 untemplated adenosines (As) in aligned sequences as evidence to determine the location of

95 poly (A) CS. Although these tools are able to discover novel poly(A) CS with high resolution, they

96 may lose sensitivity in low coverage sequencing libraries and when transcript expression is low.

97 For approaches based on expression levels, poly(A) CS prediction is a by-product of APA

98 analysis. Tools adopting this approach, which include DaPars, APAtrap, and QAPA [25-27], all rely

99 on differential expression in the 3' UTRs of matched samples. However, due to the low sequence

100 complexity of 3' UTRs and the resulting sequencing data artefacts, read coverage in these regions

101 may not reflect true expression levels, impacting differential expression analysis and associated

102 statistical tests. Consequently, these methods may fail to detect real but lowly represented APA

103 events. Moreover, they only report poly(A) CS involved in significant APA events through

104 comparative analyses, and thus these tools are not capable of profiling poly(A) CS in given

105 samples.

106 Another approach to address the prediction problem is through the use of deep learning

107 methods, and several such models have been proposed recently, such as DeeReCT-PolyA [28],

108 DeepGSR [29], and DeepPASTA [30]. For these tools, hidden features are learnt and modeled

109 directly from input nucleotide sequences. Using this approach, DeeReCT-PolyA and DeepGSR 
110 have been reported to show around $90 \%$ accuracy on test sequences $[28,29]$. Combining both

111 sequence and RNA secondary structure information, DeepPASTA, was reported to have an area

112 under the curve score over 93\% in predicting poly(A) CS. However, these tools were only bench-

113 marked on pre-constructed datasets composed of positive and negative genomic sequences, and

114 none have been applied to real experimental transcriptome data for prediction. Another

115 limitation of DeeReCT-PolyA and DeepGSR is that they were only trained to distinguish whether

116 a PAS is real. However, it is known that a small fraction (yet a substantial number) of functional

117 human poly(A) CS does not require a PAS [32,33]. At present, these approaches overlook poly(A)

118 CS with noncanonical cis-acting elements, which would likely negatively impact their

119 performance on experimental data.

120 Terminitor

121 Despite the availability of various tools, comprehensively and efficiently profiling poly(A) CS in

122 RNA-seq samples remains challenging. In this study, we present Terminitor, a deep neural

123 network (NN) model for fast and accurate poly(A) CS recognition, independent of the existence

124 of a PAS. Terminitor takes fixed length sequences as input and performs a three-label

125 classification to determine whether the sequence contains a poly(A) CS, a non-polyadenylated

126 CS, or non-CS. Coupled with Terminitor, we also propose a profiling pipeline that generates

127 prediction of poly(A) CS using raw RNA-seq data as input. The performance of this pipeline was

128 cross-validated on two datasets of sequences (describing human and mouse transcriptomes) to

129 select the optimal input sequence length and model hyperparameters. We have benchmarked

130 our pipeline against competing tools on experimental paired-end RNA-seq libraries generated

131 from the Illumina (San Diego, CA) TruSeq paired-end and Pacific Biosciences (Menlo Park, CA) 
132 Single Molecule, Real-Time (SMRT) Sequencing platforms.

\section{Results}

\section{Performance of Terminitor using different sequence lengths}

135 Terminitor is trained on experimentally validated annotated nucleotide sequences containing

136 three classes of sites: (1) poly(A) CS, (2) non-poly(A) CS, and (3) non-CS. Using cross-validated

137 optimal hyperparameters, it computes the probability of a test sequence belonging to one of the

138 three classes.

139 To train, validate, and test the model, we collated two reference datasets describing human

140 and mouse transcriptomes, based on poly(A) CS databases and Ensembl annotations. These

141 datasets are composed of labeled sequences of uniform length with three parts: upstream

142 sequence, a site from one of the three classes, and downstream sequence. Upstream sequences

143 are from corresponding transcripts for the first two classes and from genomic regions for the

144 third class, while downstream sequences are genomic, and the transition from upstream

145 sequence to downstream sequence is marked as the cleavage site or lack thereof (S2 Fig). Since

146 there is no consensus from previous work on how the input sequence length influences

147 prediction accuracy, we built models with different lengths to evaluate its effect on performance.

148 Eight upstream lengths (200, 160, 120, 100, 80, 60, 40 and $20 \mathrm{nt})$ and five downstream lengths

$149(200,100,40,20$ and $0 \mathrm{nt})$ were chosen to create a total of 40 models, which were trained to

150 explore the impact of sequence length on poly(A) CS prediction (Fig 1). In general, all models

151 show clear separation between poly(A) CS and the other two labels (S3 Fig). The accuracy of all

15240 models is above $87 \%$, including the one trained with only 20 nt long upstream sequence. As

153 expected, models trained with sequences containing both upstream and downstream sequences 
154 perform better than the ones trained with upstream sequences alone $(0 \mathrm{nt}$ downstream

155 sequence length). However, given the same upstream sequences, models trained with

156 downstream sequences longer than 20 nt perform comparably to each other. The 28 models

157 trained with upstream sequence lengths in $\{40,60,80,100,120,160,200\}$ and downstream

158 sequence lengths in $\{20,40,100,200\}$ have an average accuracy of $94.50 \% \pm 0.0015$. More

159 interestingly, longer downstream sequences do not necessarily lead to better performance.

160 Models trained with 200 nt downstream sequences have slightly lower accuracy than the ones

161 trained with shorter lengths, suggesting that some polyadenylation recognition patterns maybe

162 obscured by sequence motifs further downstream.

163 Fig 1. Model performance on human reference dataset. Each facet represents one 164 downstream sequence length, and the $x$-axis of each facet plot shows the up-stream sequence 165 length. Each data point is the mean of 5-fold cross validation and the error bar is the standard 166 deviation.

167 On the other hand, given the same downstream sequence, the performance of models

168 plateaus when the upstream sequence is $40 \mathrm{nt}$ or longer. Similarly, longer upstream sequences

169 do not guarantee better performance either, especially when the upstream length is much longer

170 than the downstream length. For example, the model with $120 \mathrm{nt}$ upstream and $20 \mathrm{nt}$

171 downstream sequences is nearly $0.5 \%$ more accurate than the one with 200 nt upstream and 20

172 nt downstream sequences. This decrease is mainly due to a drop in specificity, which means the

173 model has more false positive predictions with longer upstream sequence.

\section{Tool comparison on experimental data}

175 In order to demonstrate the application of Terminitor on poly(A) CS profiling, we designed a 176 prediction pipeline and applied it on two human RNA-seq samples, Human Brain Reference RNA 
177 (HBR) and Universal Human Reference RNA (UHR), to predict poly(A) CS from reconstructed

178 transcripts. As done previously, we trained Terminitor with 40 length combinations, applied all

17940 models on UHR sample, and computed the Pareto frontier based on sensitivity and precision.

180 The sensitivity of Terminitor is positively correlated with upstream sequence length, while there

181 is no clear trend between the downstream sequence length and the performance metrics (S4 Fig).

182 For demonstration purposes, we chose $\pm 100 \mathrm{nt}$ model for performance benchmarking and

183 comparison.

184 Profiling poly(A) CS for species without a good reference annotation can be challenging. Thus

185 it is interesting to test whether the information learnt from one species is transferable to another.

186 In addition to the model built on the above-mentioned human reference dataset, we also

187 generated a model that was separately trained on the mouse dataset. We applied these two

188 models on the UHR and HBR samples and compared the performance with KLEAT, DeepPASTA,

189 DeeReCT-PolyA and DeepGSR (Fig 2A, S5A Fig). Poly(A) CS predicted by these methods were

190 compared against Ensembl annotation to calculate the sensitivity and precision. Note that the

191 denominator in sensitivity calculations represents all Ensembl annotations, yet for a given sample

192 not all transcripts would be expressed. Without a priori information on gene and isoform

193 expression, this is the most appropriate solution at it penalizes all methods the same way,

194 preserving their relative performance for fair comparisons.

Fig 2. Performance comparison on the UHR sample. A. Sensitivity and specificity of poly(A) 196 CS predictions from Terminitor, KLEAT, DeepPASTA, DeeReCT-PolyA and DeepGSR on Ensembl 197 annotated poly(A) CS. The two pre-trained models of DeepGSR are the one with sequences 198 containing only the strongest PAS hexamer AATAAA, and the one with sequences containing 16 199 hexamer motifs pooled together. The two pre-trained models of DeeReCT-PolyA are derived from 200 the dragon dataset and omni dataset as described (Xia et al., 2019). Two pre-trained Terminitor 201 models are derived from the human and mouse datasets. The navy/blue crosses on Terminitor 
202 human/mouse model represent probability $=0.5$ cut-off, respectively. B. Percentage of poly(A) 203 CS that are missing from Ensembl annotation are supported/not supported by PolyA-Seq. We 204 used probability $=0.5$ cut-off for Terminitor and DeepPASTA predictions.

205 We also note that DeeReCT-PolyA and DeepGSR are binary classifiers, while Terminitor 206 outputs a probability value that can be tuned in favour of either sensitivity or precision.

207 Nevertheless, Terminitor trained on human sequences consistently outperforms all competing

208 tools in both metrics, except for one data point, which corresponds to the raw, unfiltered KLEAT

209 predictions. However, the precision of raw KLEAT predictions is arguably too low (35.90\% and

$21025.52 \%$ for UHR and HBR samples, respectively) to be used for high-confidence profiling.

211 Although KLEAT performs comparably to Terminitor on UHR sample, its performance is

212 surprisingly low on HBR sample regardless of what read evidence is used to filter its raw

213 predictions. It is also worth mentioning that the four models built by DeeReCT-PolyA and

214 DeepGSR all have a precision lower than 50\%, while precision of the Terminitor human model (at

215 probability $=0.5$ cut-off) is $55.59 \%$ and $57.72 \%$ for UHR and HBR samples, respectively.

216 Since a given sample may express novel transcripts or transcripts with novel poly(A) CS, we

217 compared the falsely predicted $\operatorname{poly}(\mathrm{A})$ CS determined by Ensembl annotation with the

218 corresponding PolyA-seq predictions to identify putative novel events with PolyA-seq evidence

219 (Fig 2B, S5B Fig). Although Terminitor did not predict the highest number of high confidence

220 poly $(A)$ CS overall, it is always the pipeline having the highest percentage $(35.45 \%$ and $36.95 \%$ for

221 UHR and HBR sample, respectively) of predictions that have PolyA-seq evidence support. This

222 suggests its high precision in detecting novel poly(A) CS when compared to the other methods.

223 Although the raw KLEAT predictions identified a large number of poly(A) CS, only a small

224 proportion can be verified by PolyA-seq. We observed that to maintain precision, certain filters 
225 have to be applied to make use of KLEAT predictions, while the choice of filter may be highly

226 library-dependent.

227 We compared the runtime and peak memory usage of all competing tools under the same

228 computing environment (Table 1). All five pipelines used RNA-Bloom [34] as the de novo RNA-

229 seq assembler to start with, and the peak memory usage of all pipelines is the same (37.87 GB),

230 contributed by RNA-Bloom. Terminitor, DeepPASTA, DeepGSR, and DeeReCT-PolyA shared the

231 same pipeline to identify candidate sequences, and the only difference is the NN used for

232 sequence classification, which resulted in runtime differences. With a runtime of 7:06:52 hours,

233 DeeReCT-PolyA is the fastest tool, followed by Terminitor with less than 5-minute (1.17\%) run

234 time difference. DeepPASTA took two hours more than the first two competitors because of the

235 RNA structure prediction step, although this extra step did not help improve its prediction

236 performance. The long runtime of DeepGSR is probably due to its implementation, which was

237 only compatible with Python 2.7 and older versions of Keras and Theano. KLEAT is more than

238 three times slower compared to all other tools because it involves computationally expensive

239 read-to-contig and contig-to-genome alignments.

Table 1 Runtime comparison. The runtime for all four pipelines includes de novo assembly of UHR sample (two replicates), alignments, candidate poly(A) CS identification and classification.

\begin{tabular}{l|cc}
\hline Pipeline & Wallclock Runtime (hh:mm:ss) & Wallclock Runtime without assembly \\
DeeReCT-PolyA & $7: 06: 52$ & $17: 29$ \\
Terminitor & $7: 11: 23$ & $22: 00$ \\
DeepPASTA & $9: 17: 49$ & $2: 28: 26$ \\
DeepGSR & $11: 10: 22$ & $4: 20: 59$ \\
KLEAT & $25: 17: 00$ & $18: 27: 37$ \\
\hline
\end{tabular}


The Ensembl annotation is a repertoire of transcript annotations from multiple tissues and cell

243 lines in a range of biological states, so not all of the annotated poly(A) CS are expressed in the

244 HBR and UHR samples, as noted above. We quantified the expression levels of all polyadenylated

245 reference transcripts in Ensembl, and then examined how the four tools perform with respect to

246 poly(A) CS at different expression levels (Fig 3, S6 Fig). Aside from the spikes corresponding to

247 KLEAT raw predictions, Terminitor consistently performs better than all competitors in four tiers

248 of expression thresholds. As expected, the sensitivity of all tools increases as the expression level

249 threshold increases, because unexpressed transcripts are removed from the denominator. The

250 performance difference between Terminitor and other tools increases as well when the

251 expression cut-off increases. In fact, the performance metrics of Terminitor mouse model on

$252 \operatorname{poly}(\mathrm{A})$ CS expressed at $>20$ TPM are similar to, or better than, DeepPASTA, DeepGSR and

253 DeeReCT-PolyA models for both UHR and HBR samples, showing the promising application of

254 Terminitor on species without a good reference transcriptome annotation.

Fig 3. Performance comparison on the UHR sample with different expression level cut-offs.

256 Sensitivity and precision of poly(A) CS predictions of Terminitor, KLEAT, DeepPASTA, DeeReCT-

257 PolyA and DeepGSR on expressed Ensembl transcripts. The four facet plots represent the

258 comparison between all expressed transcripts, expressed transcript with TPM $>1,>10$, and $>20$,

259 as indicated.

260 Single nucleotide variation in PAS affects APA choices

261 One of the advantages of Terminitor is that it is capable of predicting poly(A) CS solely based

262 on sequence context. Consequently, poly(A) CS created or destroyed by base changes can also

263 be identified. We carefully examined all candidate sequences belonging to the UHR sample, and

264 identified sequences containing different PAS hexamer motifs from the reference human

265 genome assembly. Through comparisons between the predicted probabilities and the base 
variation, we discovered two cases of variation-associated polyadenylation.

267 The first variant base is recorded in dbSNP as rs6484833, a C-to-T change at chr11:36273982

268 (GRCh38.p12) on the reverse strand as seen in Integrative Genomics Viewer (S7A Fig) [35,36].

269 This polymorphism creates the strongest hexamer AATAAA on gene COMMD9, and it is

270 associated with an unannotated poly(A) CS at chr11:36273960 on the terminus exon. We

271 speculate that this association may be causal, the former causing the latter. In our pipeline, RNA-

272 Bloom successfully reconstructed this novel transcript to full length with a poly(A) tail, and

273 Terminitor predicted it as a poly(A) CS with 99\% probability. This poly(A) CS is also detected in

274 PolyA-Seq of the same sample. Based on Ensembl annotation, COMMD9 has three poly $(\mathrm{A}) \mathrm{CS}$

275 associated with the same terminus exon. This site results in the second-longest 3' UTR, which

276 harbours three miRNA binding sites and extensive RNA binding protein (RBP) sites for 19 RBPs

277 (S8 Fig).

278 The second variant base is rs15342, a T-to-C change at chr15: 101070089 on gene LRRK1 (S7B

279 Fig). This polymorphism destroys the strongest hexamer AATAAA, which is associated with an

280 annotated poly(A) CS 22 nt downstream. There are 21 sequencing reads containing this

281 polymorphism extend beyond this poly $(A)$ CS, while reads carrying the wildtype are all

282 polyadenylated. RNA-Bloom successfully assembled both the wildtype transcript with a poly(A)

283 tail and the variant transcript without poly(A) tail. Terminitor classifed the wildtype and variant

284 transcripts to contain poly(A) CS with a probability of $98 \%$ and $1 \%$, respectively. This

285 polymorphism may prevent cleavage at the poly $(A)$ CS immediately downstream, leading to the

286 alternative usage of the distal poly(A) CS, and producing a transcript with longer 3' UTR. Similarly,

287 extensive (16) miRNA binding sites and RBP sites for 77 RPBs are also predicted to exist on this 
extended 3' UTR (S9 Fig).

\section{Terminitor performance on long reads}

290 Although the current short read sequencing followed by de novo transcriptome assembly is

291 part of Terminitor's mainstream RNA-seq analysis pipeline, the advent of long read sequencing

292 technologies provides unprecedented opportunity to study APA at the transcript isoform level.

293 Here we applied the Terminitor pipeline on two sequence libraries from the human sample

294 NA12878, namely Illumina short reads and PacBio circular consensus sequence (CCS) reads (see

295 Methods for details). Terminitor consistently profiles three times or more poly(A) CS in the PacBio

296 library as in the Illumina library, even under different expression level cut-offs (Fig 4). However,

297 with the Illumina library, Terminitor is able to detect more poly(A) CS at a higher precision (>

298 70\%). As the probability cut-off becomes more stringent, less positive poly(A) CS are predicted

299 by both libraries, but the overlapping percentage with respect to the Illumina library increases

300 (S10 Fig). For predicted poly(A) CS with a probability $>90 \%, 59.27 \%$ of the CSs found in the

301 Illumina library are also found in the PacBio library.

Fig 4. Performance comparison between PacBio library and Illumina library on a $\boldsymbol{H}$. sapiens NA12878 sample. Sensitivity and precision of poly(A) CS predicted by Terminitor on PacBio and

304 Illumina libraries at different expression level cutoffs. The four facet plots represent the 305 comparison between all expressed transcripts, expressed transcript TPM $>1,>10$, and $>20$, as 306 indicated.

\section{Discussion and Conclusions}

308 Here we introduce a novel deep learning model named Terminitor to detect and classify 3' UTR 309 cleavage sites with or without poly $(\mathrm{A})$ tails, and a profiling pipeline that starts from raw RNA-seq 310 reads to characterize expressed poly(A) CS. The novelties of our model and contributions in this 311 work are as follows: 
1. The use of concatenated $k$-mer representations instead of raw sequence as model input.

313 Previous applications of deep learning in omics research have shown that designing a proper

314 representation of the raw data is critical to facilitate classification performance. Inputting

315 additional information, namely well-studied sequence features, can also alleviate the burden of

316 learning complex structures. In the case of polyadenylation, several cis-acting elements have

317 been revealed, such as the GU-rich sequences in the downstream, UGUA elements in the

318 upstream, and the conserved hexamer motif. Inspired by the usage of $k$-mer in the

319 genome/transcriptome assembly tasks, Terminitor uses $k$-mer representations of raw sequences

320 to mimic functional motifs. Since the auxiliary elements may have different lengths, multiple $k$ -

321 mer representations are jointly used to, and are observed to push the accuracy higher.

2. The use of an embedding layer to learn the similarities between $k$-mer pairs. One-hot

323 encoded $k$-mer representation of raw sequence is high dimensional and sparse. In our case, the

324 input dimension is exponential to $k$, and most positions in the input vector are zeros. This is

325 computationally inefficient, and would not capture similarities between $k$-mers. To address this,

326 we borrowed the concept of word embedding from natural language processing and used it as

327 an interface between the input layer and the subsequent hidden layers. Essentially, in the

328 embedding layer, one-hot encoded $k$-mers are converted into dense vectors, representing the

329 projection of $k$-mers in a continuous vector space. Consequently, motifs with similar functions

330 are likely to have similar weights, and treated similarly in the subsequent layers.

331 3. Separation of non-polyadenylated CSs from polyadenylated CSs. To our knowledge,

332 Terminitor is the first poly(A) CS classifier that includes non-polyadenylated CS label. Although

333 most protein-coding and long-noncoding RNAs contain poly(A) tails at their 3' ends, a significant 
334 number of functional transcripts are not polyadenylated [37]. Since the 3' end processing

335 mechanism of this group is distinct from polyadenylated CS group, separating it out as a new

336 class improves the performance of the model. Sequence clustering based on Terminitor's last

337 hidden layer also groups sequences containing non-polyadenylated CS and non-CS together as a

338 big cluster (S3 Fig).

339 Compared to targeted $3^{\prime}$ end sequencing technologies, RNA-seq data, whether generated

340 using short or long read techniques, are more accessible and widely used in transcriptome studies.

341 In the reported work, we demonstrate that the Terminitor pipeline can identify poly(A) CS on

342 expressed transcripts with high precision in comparison to the state-of-the-art methods for RNA-

343 seq data. Failure in detecting a real and expressed poly(A) CS is mostly due to the failure of

344 reconstructing transcripts to their $3^{\prime}$ ends. For instance, in the UHR sample, among the 58,925

345 expressed poly(A) CS that were not detected, more than half $(30,181)$ have a TPM less than one,

346 and the majority of them have very few or no reads supporting the poly $(A)$ tail because of

347 sequencing bias.

348 We observed that false positive predictions by Terminitor generally occur for three reasons.

349 The first and most common scenario is mis-assemblies due to the low sequence complexity of

350 the 3' UTR. Second, these poly(A) CS are in fact real novel events, but unannotated; around 30\%

351 of the Terminitor false positives (using the Ensembl annotation) are corroborated by PolyA-Seq

352 evidence (Fig 2B, S5B Fig). Third, mis-alignment of upstream sequences, meaning the sequence

353 may contain a real poly(A) CS, but the genomic location is incorrect. In the Terminitor pipeline,

354 when an upstream sequence is multi-mapped, only the primary alignment is chosen to help

355 reduce the false positive rate. However, when the sequence comes from gene paralogs, the 
genomic location inferred from the chosen alignment does not necessarily correspond to that of

357 the expressed paralog.

In principle, the Terminitor pipeline can be applied on any reconstructed transcripts to determine if its $3^{\prime}$-end contains a poly(A) CS, or non-polyadenylated CS, or no site (3' incomplete).

Aside from benchmarking its performance on traditional Illumina short read assemblies, we also twice the CS prediction sensitivity when using the PacBio vs. Illumina library, probably because more sequences containing real poly(A) CS are fed into the NN model. PacBio CCS reads are selfcorrected through consensus calling step, resulting in near- or full-length transcripts with poly(A)

365 tails. In contrast, transcripts reconstructed from an Illumina library may suffer from misassemblies, and lowly expressed poly(A) CS may be obscured by longer transcripts. In our analysis,

367 among the 548,448 candidate sequences extracted from the PacBio library, $29.71 \%$ of them 368 contain untemplated poly(A)s, while the ratio is much lower (7.22\%) in 381,934 assembled 369 sequences using the Illumina library.

In summary, Terminitor outperforms all state-of-the-art methods in the datasets we

371 benchmarked them on, and executes within a reasonable time, making it an attractive tool to

372 complement other RNA-seq analysis pipelines. The performance of Terminitor is robust on both

373 short and long-read sequencing technologies. As long-read RNA-seq finds broader uptake in

374 transcriptome studies, it is poised to shed light on the association between poly(A) CS and

375 transcript isoforms, fueling a new era of APA research. Terminitor is a candidate enabling 376 technology for such studies. Further, we have observed how Terminitor can identify novel poly(A) 377 CS caused by base variations. As has been elucidated in previous studies, novel poly(A) CS are 
378 expressed in cancer tissues, and with the help of Terminitor, mechanisms and impacts of APA can

379 be better understood. Even for species without an established poly(A) CS database, their 380 expressed poly(A) CS can be identified with Terminitor using transfer learning, where the model

381 is trained on a different but related species. The usage of Terminitor, as a sequence classifier, is

382 not limited to poly(A) CS profiling, but can also be extended to facilitate gene annotation and

383 testing the completeness of assembled transcripts. We expect Terminitor to have broad

384 applications in genome/transcriptome annotation, novel isoform identification, APA analysis and 385 gene regulation studies.

\section{Methods}

\section{Datasets}

388 To train and validate the NN model, and to demonstrate transfer learning, we collated two 389 reference datasets describing human and mouse transcriptomes, based on poly $(A)$ CS databases

390 and Ensembl annotations. Each dataset is composed of sequences with equal lengths coming

391 from three classes: poly(A) CS, non-poly(A) CS, and non-CS (Table 2). PolyA_DB version3.1 was 392 used as our primary poly(A) CS library [13]. We first mapped poly(A) CS recorded in PolyA_DB3 393 to Ensembl annotation release 94 using bedtools closest (v2.27.1) and selected the most 394 compatible transcript for each site. Poly(A) CS supported by less than 1 Reads Per Million (RPM) 395 or located more than $1000 \mathrm{nt}$ downstream of any annotated transcript were considered 396 unreliable and were discarded.

Table 2. Size and origin of datasets for model training, testing, and validation

\begin{tabular}{llll}
\hline Species & poly(A) CS & non-poly(A) CS & non-CS \\
\hline H. sapiens & 52,457 & 27,595 & 52,460 \\
M. musculus & 75,661 & 37,028 & 75,660 \\
\hline
\end{tabular}


To avoid potential poly(A) CS contaminating the other two classes, we expanded our poly(A)

399 CS library by incorporating three additional poly $(A)$ databases, including APADB v2, APASdb, and

400 PolyASite. Again, we used bedtools closest to assign poly(A) CS in the compiled poly(A)

401 library to Ensembl transcripts. Transcripts with no poly(A) CS from the above-mentioned

402 databases assigned were considered as cleaved but not polyadenylated, and their $3^{\prime}$ ends

403 constituted the non-polyadenylated CS set.

$404 \quad$ Non-CS comprises five types of genomic locations: exonic, intronic, intergenic, 5' and 3'

405 untranslated regions, and are randomly selected at least 100 nt away from any annotated 3' ends,

406 including Ensembl annotation and compiled poly(A) library.

407 Our datasets are imbalanced in their label abundances due to the limited number of non-

408 polyadenylated CS. However, to fully exploit the potential of deep NNs, we used all non-

409 redundant poly(A) CS and CS generated as above descriptions, and deliberately matched the

410 number of pseudo sites to the number of poly(A) CS. Because the label abundances are

411 imbalanced, we used both accuracy and the F-measure, the harmonic mean of precision and

412 recall, as assessment metrics during model training and benchmarking.

\section{Model training}

414 The NN model is implemented in Python 3.6.6, using Keras library 2.2.4 [38] with TensorFlow

4151.11 .0 [39] as the backend. To evaluate the performance of the model with different

416 hyperparameter combinations and input sequence lengths, we used stratified 5-fold cross

417 validation on the whole dataset to monitor the overall accuracy of three labels, and accuracy,

418 sensitivity, and specificity on poly $(A)$ label. All performance metrics on the training sets reported 
419 in this study are the mean and standard deviation of five validation runs. In each run, $80 \%$ of data

420 is used for training and $20 \%$ is used for validation. Next, we trained the model using the entire

421 training set and used it for benchmarking the performance on experimental data.

\section{Model architecture and hyperparameter tuning}

423 Searching for the optimal hyperparameters for a deep NN has always been a tedious task, as

424 many parameters are dependent on each other in the way they influence performance. We first

425 built the basic frame with one input layer, one embedding layer, and one output classification

426 layer, and then fine-tune hyperparameters related to architecture and training using grid search,

427 as listed in Table 3. For all models, initial model weights were randomly drawn from Glorot

428 uniform initializer [40]. The weights of the three neurons in the output layer are computed via

429 softmax activation function, representing the probability distribution of the three labels, and they

430 always add up to one. To avoid over-fitting, we applied early stopping in training each model. As

431 an iterative method, the validation loss is monitored at each training epoch, and when it stays

432 the same or increases for the next ten epochs, the training process is stopped. Without early

433 stopping, too many training epochs will lead to nearly $100 \%$ accuracy on training set, yet yield

434 very low accuracy on validation and test sets.

Table 3 Model hyperparameter tuning. Bolded parameters are the selected optimal values

\begin{tabular}{lll}
\hline Parameter type & Parameter & Search space \\
\hline Training & Optimizer & SGD, Adam, Adagrad, RMSprop \\
\cline { 2 - 3 } & Learning rate & $0.1,0.01, \mathbf{0 . 0 0 1}, 0.0001$ \\
\cline { 2 - 3 } & Loss function & Categorical_cross_entropy, mean_squared_error \\
\cline { 2 - 3 } K-mer representation & $\{1\},\{2\},\{3\},\{4\},\{5\},\{6\},\{7\},\{8\},\{9\},\{10\},\{11\},\{12\}$, \\
& $\{4,5\},\{4,6\},\{4,8\},\{4,10\},\{4,11\},\{4,5,6\},\{4,6$, \\
& $8\},\{4,5,6,10\},\{4,6,8,10\},\{4,5,6,8,10\}$ \\
\hline & $32,64,128,256$ \\
\hline
\end{tabular}




\begin{tabular}{|c|c|c|}
\hline \multirow[t]{6}{*}{ Architecture } & Number of layers & $1,2,3,4,5$ \\
\hline & Number of nodes for & $4,16,32,64,128,256,512,1024$ \\
\hline & layers & (Refer to Fig 6 for details) \\
\hline & Regularization & Dropout: $\mathbf{0}, 0.1,0.2,0.5$ \\
\hline & $\begin{array}{l}\text { Activation function for } \\
\text { hidden layers }\end{array}$ & $\tanh , \mathbf{R e L U}$ \\
\hline & $\begin{array}{l}\text { Activation function for } \\
\text { output layer }\end{array}$ & Softmax, Sigmoid \\
\hline
\end{tabular}

The final architecture of Terminitor is depicted in Fig 5 and the selected optimal

$$
l=\sum_{k \in\{4,6,8,10\}} 4^{k}
$$

Fig 5. Architecture of Terminitor. The information flows from bottom up. During training stage, reference transcripts are aligned back to the reference genome, and flanking sequence on both 442 side of the poly(A) CS is extracted (grey thick sequence). The sequence is $k$-merized and four $k$ 443 values are used. Then all $k$-mer representations are concatenated and each $k$-mer is one-hot 444 encoded (each square represents one $k$-mer). The input sequences are fed into an embedding 445 layer, followed by three fully connected layers, and then the probability of belonging to one of 446 the three classes is calculated through a Softmax function.

447 Then an embedding layer with 128 nodes is used to connect the input layer and hidden layers.

448 The next four layers are all fully connected, and the activation function between each adjacent

449 layer is the rectified linear unit (ReLU) function. Since dropout regularization did not achieve 450 better performance, it was not performed in the final model.

\section{Experimental data}


452 The raw RNA-seq reads for the two MicroArray Quality Control project reference human samples,

453 HBR and UHR, were downloaded from Illumina BaseSpace, and each sample has two replicates

454 (https://basespace.illumina.com/projects/3777775/samples) [41]. RNA-seq libraries of the four

455 samples were prepared with Illumina TruSeq Stranded kits using poly(A) selection, and

456 sequenced as 75bp paired-end reads. The Illumina and PacBio reads for NA12878 were obtained

457 from an earlier study under accession number SRR1153470 and SRR1163655, respectively [42].

458 The Illumina reads are from a poly(A) selected library sequenced with 75 bp stranded paired-end

459 reads. The PacBio library was prepared from the same batch of poly(A) selected RNA and

460 sequenced with PacBio CCS method. The filtered PolyA-Seq sites were downloaded from the NCBI

461 Gene Expression Omnibus (GEO) under study GSE30198. We combined sites detected by the two

462 HBR replicates (GSM747473 and GSM GSM747474), and sites detected by the two UHR replicates

463 (GSM747475 and GSM747476) together respectively for benchmarking analysis.

\section{Prediction pipeline}

465 We also provide a poly(A) CS prediction pipeline based on Terminitor. When using Illumina short

466 reads, the pipeline starts by assembling RNA-seq data using RNA-Bloom [34]. RNA-Boom is run

467 with the option -stratum 01, which allows extension of all fragments regardless of its

468 coverage, and the option --polya, which prioritizes the assembly of transcripts with poly(A)

469 tails. The false positive rate (FPR) of Bloom filters in the program is set to 0.005 . Then, all

470 assembled transcripts are aligned to the reference genome hg38 and compared to Ensembl

471 annotation release 94 to select sequences with untemplated As at their $3^{\prime}$ ends, or sequences

472 that end in the $3^{\prime}$ UTR of annotated transcripts. Similar to the structure of sequences in the

473 training dataset, selected sequences are used to construct test sequences, each composed of 
474 three parts: a fixed length (100 nt) of upstream sequence extracted from the assembled

475 transcript, a potential cleavage site, and a fixed length (100 nt) of downstream genomic sequence

476 extracted from the reference. Finally, these test sequences are fed into the trained Terminitor

477 model for classification.

\section{Evaluation of prediction pipeline}

479 Poly $(A)$ CS are often observed as a group of neighboring sites rather than a precise genomic

480 location [7]. To accommodate, predicted poly(A) CS within $30 \mathrm{nt}$ of each other are clustered, and

481 the one with the highest probability score is chosen. Ensembl annotated poly(A) CS are also

482 clustered in the same way, and the one located in the median position is chosen as the

483 representative. Then, the predicted list is compared with the annotated list to compute hits and

484 misses. In our evaluation system, a true positive poly(A) CS is a predicted one that lies within \pm

$48530 \mathrm{nt}$ of an annotated site; a false positive site is a predicted poly $(\mathrm{A}) \mathrm{CS}$ that does not lie within \pm

$48630 \mathrm{nt}$ of any annotated ones; false negative means an annotated site does not have any predicted

487 site within \pm 30 nt. For this analysis, true negative (no real or predicted poly(A) CS in a certain

488 location) is not informative for experimental data. Using these definitions, we report sensitivity

489 and precision as performance measures for all experimental data tests. Note that in the training

490 data, true negative is informative, and we also report accuracy for our cross validation results.

\section{Tool comparison}

492 We applied the proposed prediction pipeline on experimental data and compared its

493 performance with the performance of the state-of-the-art methods. All experiments were run on

494 Centos 6.7 system with 12 Intel Xeon E5-2650 CPUs and 84 GB memory. 
KLEAT pipeline was chosen as a representative of read-evidence based approaches. The auxiliary files KLEAT uses include: 1) RNA-seq assemblies from RNA-Bloom, 2) read to contig

497 alignment files generated by BWA-MEM 0.7.17-r1188 [43], and 3) contig to genome alignment

498 files generated by GMAP version 2017-01-14 [44]. Since KLEAT only works for the human genome 499 reference hg19, all predicted poly(A) CS were converted into hg38 genomic coordinates by UCSC 500 liftOver [45]. KLEAT outputs all possible poly(A) CS and their supporting evidence, such as the 501 length of poly $(A)$ tail in reconstructed transcripts, the number of reads containing poly $(A)$ tail, 502 and the length of $\operatorname{poly}(\mathrm{A})$ tail of reads that can be mapped to a reconstructed transcript. We 503 applied filters to the raw predictions with different combinations of three evidence types and 504 computed the Pareto frontier on the sensitivity-precision plane.

505 Two recent deep learning models, DeepGSR and DeeReCT-PolyA, were also included in the comparison. Like Terminitor, these two tools are NN models expecting fixed length sequences as

507 input. Starting from all candidate sequences processed from our prediction pipeline, we selected

508 only the ones containing PAS as these tools expects PAS in the input sequences. Then we adjusted 509 the sequence lengths as required by the two models ( $\pm 300 \mathrm{nt}$ around the PAS for DeepGSR; \pm $510100 \mathrm{nt}$ around the PAS for DeeReCT-PolyA), and fed into these two models for classification. For

511 DeepGSR, we used two pre-trained models from its publication, one only on the AATAAA 512 hexamer, and the other on all 16 hexamers [29]. Similarly, the DeeReCT-PolyA package provides 513 two pre-trained models based on two human datasets, dragon and omni, both of which were 514 included in the comparison. Both datasets were generated by previous poly $(\mathrm{A})$ prediction 515 researches $[46,47]$. Both DeepGSR and DeeReCT-PolyA only output a binary value instead of a 
confidence score that can be tuned with different cut-offs, so their predictions contributed to

517 four data points in the comparison plot: two models for two tools.

\section{Data access}

519 Terminitor is implemented using Python 3.6 with Keras package. The software package is freely

520 available under GNU General Public Licence. The source code and manual are available at our

521 GitHub repository https://github.com/bcgsc/Terminitor. Previously published data used in this

522 work are described in the Methods section, and are available from several public repositories.

523 The human and mouse reference datasets colated in this study and trained models are deposited

524 in public ftp site http://www.bcgsc.ca/downloads/supplementary/Terminitor.

\section{Reference}

1. Edmonds $\mathrm{M}$, Vaughan $\mathrm{MH}$, Nakazato H. Polyadenylic acid sequences in the heterogeneous nuclear RNA and rapidly-labeled polyribosomal RNA of HeLa cells: possible evidence for a precursor relationship. Proc Natl Acad Sci U S A. 1971;68: 1336-40. doi:10.1073/pnas.68.6.1336

2. Neve J, Patel R, Wang Z, Louey A, Furger AF. Cleavage and polyadenylation: Ending the message expands gene regulation. RNA Biol. 2017;14: 865-890.

3. Barrett LW, Fletcher S, Wilton SD. Regulation of eukaryotic gene expression by the untranslated gene regions and other non-coding elements. Cellular and Molecular Life Sciences. 2012. pp. 3613-3634. doi:10.1007/s00018-012-0990-9

4. Shi Y, Chan S, Martinez-Santibañez G. An up-close look at the pre-mRNA 3'-end processing complex. RNA Biol. 2009;6: 522-525. doi:10.4161/rna.6.5.9554

5. Zhang H, Lee JY, Tian B. Biased alternative polyadenylation in human tissues. Genome Biol. 2005;6: R100. doi:10.1186/gb-2005-6-12-r100

6. Tian B, Graber JH. Signals for pre-mRNA cleavage and polyadenylation. Wiley Interdisciplinary Reviews: RNA. 2012. pp. 385-396. doi:10.1002/wrna.116

7. Tian $\mathrm{B}, \mathrm{Hu} J$, Zhang $\mathrm{H}$, Lutz CS. A large-scale analysis of mRNA polyadenylation of human and mouse genes. Nucleic Acids Res. 2005;33: 201-212. doi:10.1093/nar/gki158

8. Lianoglou S, Garg V, Yang JL, Leslie CS, Mayr C. Ubiquitously transcribed genes use alternative polyadenylation to achieve tissue-specific expression. Genes Dev. 2013;27: 2380-2396. doi:10.1101/gad.229328.113

9. Lembo A, Di Cunto F, Provero P. Shortening of 3'UTRs correlates with poor prognosis in breast and lung cancer. PLoS One. 2012;7. doi:10.1371/journal.pone.0031129

10. Li L, Wang D, Xue M, Mi X, Liang Y, Wang P. 3'UTR shortening identifies high-risk cancers with targeted dysregulation of the ceRNA network. Sci Rep. 2015;4: 5406. doi:10.1038/srep05406

11. Xue Z, Warren RL, Gibb EA, MacMillan D, Wong J, Chiu R, et al. Recurrent tumor-specific regulation of alternative polyadenylation of cancer-related genes. BMC Genomics. 2018;19. doi:10.1186/s12864-018-4903-7 
12. Aken BL, Ayling S, Barrell D, Clarke L, Curwen V, Fairley S, et al. The Ensembl gene annotation system. Database. 2016;2016: baw093. doi:10.1093/database/baw093

13. Wang R, Nambiar R, Zheng D, Tian B. PolyA-DB 3 catalogs cleavage and polyadenylation sites identified by deep sequencing in multiple genomes. Nucleic Acids Res. 2018;46: D315-D319. doi:10.1093/nar/gkx1000

14. Liu D, Brockman JM, Dass B, Hutchins LN, Singh P, McCarrey JR, et al. Systematic variation in mRNA 3'-processing signals during mouse spermatogenesis. Nucleic Acids Res. 2007;35: 234-246. doi:10.1093/nar/gkl919

15. Mayr C. Evolution and Biological Roles of Alternative 3'UTRs. Trends in Cell Biology. 2016. pp. 227-237. doi:10.1016/j.tcb.2015.10.012

16. Shepard PJ, Choi E-A, Lu J, Flanagan LA, Hertel KJ, Shi Y. Complex and dynamic landscape of RNA polyadenylation revealed by PAS-Seq. RNA. 2011;17: 761-772. doi:10.1261/rna.2581711

17. Harrison PF, Powell DR, Clancy JL, Preiss T, Boag PR, Traven ANA, et al. PAT-seq : a method to study the integration of $3^{\prime}$-UTR dynamics with gene expression in the eukaryotic transcriptome. Rna. 2015;21: 1502-1510. doi:10.1261/rna.048355.114.

18. Chang H, Lim J, Ha M, Kim VN. TAIL-seq: Genome-wide determination of poly(A) tail length and 3' end modifications. Mol Cell. 2014;53: 1044-1052. doi:10.1016/j.molcel.2014.02.007

19. Derti A, Garrett-Engele P, Maclsaac KD, Stevens RC, Sriram S, Chen R, et al. A quantitative atlas of polyadenylation in five mammals. Genome Res. 2012;22: 1173-1183. doi:10.1101/gr.132563.111

20. Müller S, Rycak L, Afonso-Grunz F, Winter P, Zawada AM, Damrath E, et al. APADB: a database for alternative polyadenylation and microRNA regulation events. Database (Oxford). 2014;2014. doi:10.1093/database/bau076

21. You L, Wu J, Feng Y, Fu Y, Guo Y, Long L, et al. APASdb: A database describing alternative poly(A) sites and selection of heterogeneous cleavage sites downstream of poly(A) signals. Nucleic Acids Res. 2015;43: D59-D67. doi:10.1093/nar/gku1076

22. Gruber AJ, Schmidt R, Gruber AR, Martin G, Ghosh S, Belmadani M, et al. A comprehensive analysis of $3^{\prime}$ end sequencing data sets reveals novel polyadenylation signals and the repressive role of heterogeneous ribonucleoprotein C on cleavage and polyadenylation. Genome Res. 2016;26: 1145-1159. doi:10.1101/gr.202432.115

23. Birol I, Raymond A, Chiu R, Nip KMi, Jackman SD, Kreitzman M, et al. Kleat: Cleavage Site Analysis of Transcriptomes. Biocomput 2015. 2014; 347-358. doi:10.1142/9789814644730_0034

24. Bonfert T, Friedel CC. Prediction of poly(A) sites by poly(A) read mapping. PLoS One. 2017;12. doi:10.1371/journal.pone.0170914

25. Xia Z, Donehower LA, Cooper TA, Neilson JR, Wheeler DA, Wagner EJ, et al. Dynamic analyses of alternative polyadenylation from RNA-seq reveal a 3'-UTR landscape across seven tumour types. Nat Commun. 2014;5: 5274. doi:10.1038/ncomms6274

26. Ye C, Long Y, Ji G, Li QQ, Wu X. APAtrap: Identification and quantification of alternative polyadenylation sites from RNA-seq data. Bioinformatics. 2018;34: 1841-1849. doi:10.1093/bioinformatics/bty029

27. Ha KCH, Blencowe BJ, Morris Q. QAPA: A new method for the systematic analysis of alternative polyadenylation from RNA-seq data. Genome Biol. 2018;19. doi:10.1186/s13059-018-1414-4

28. Xia Z, Li Y, Zhang B, Li Z, Hu Y, Chen W, et al. DeeReCT-PolyA: A robust and generic deep learning method for PAS identification. Bioinformatics. 2019;35: 2371-2379.

doi:10.1093/bioinformatics/bty991

29. Kalkatawi M, Magana-Mora A, Jankovic B, Bajic VB. DeepGSR: an optimized deep-learning structure for the recognition of genomic signals and regions. Bioinformatics. 2018. doi:10.1093/bioinformatics/bty752

30. Arefeen A, Xiao X, Jiang T. DeepPASTA: deep neural network based polyadenylation site analysis. 
600

601

602

603

604

605

606

607

608

609

610

611

612

613

614

615

616

617

618

619

620

621

622

623

624

625

626

627

628

629

630

631

632

633

634

635

636

637

638

639

640

641

642

643

644

645

646

Bioinformatics. 2019. doi:10.1093/bioinformatics/btz283

31. Min S, Lee B, Yoon S. Deep learning in bioinformatics. Briefings in bioinformatics. 2017. pp. 851869. doi:10.1093/bib/bbw068

32. Venkataraman K, Brown KM, Gilmartin GM. Analysis of a noncanonical poly(A) site reveals a tripartite mechanism for vertebrate poly(A) site recognition. Genes Dev. 2005;19: 1315-1327. doi:10.1101/gad.1298605

33. Nunes NM, Li W, Tian B, Furger A. A functional human Poly(A) site requires only a potent DSE and an A-rich upstream sequence. EMBO J. 2010;29: 1523-1536. doi:10.1038/emboj.2010.42

34. Nip KM, Chiu R, Yang C, Chu J, Mohamadi H, Warren RL, et al. RNA-Bloom provides lightweight reference-free transcriptome assembly for single cells. bioRxiv. 2019; 701607.

doi:10.1101/701607

35. Smigielski EM. dbSNP: a database of single nucleotide polymorphisms. Nucleic Acids Res.

2000;28: 352-355. doi:10.1093/nar/28.1.352

36. Thorvaldsdóttir H, Robinson JT, Mesirov JP. Integrative Genomics Viewer (IGV): Highperformance genomics data visualization and exploration. Brief Bioinform. 2013;14: 178-192. doi:10.1093/bib/bbs017

37. Yang L, Duff MO, Graveley BR, Carmichael GG, Chen LL. Genomewide characterization of nonpolyadenylated RNAs. Genome Biol. 2011;12. doi:10.1186/gb-2011-12-2-r16

38. Chollet F. Keras: Deep Learning for humans. In: Github [Internet]. 2015. Available: https://github.com/keras-team/keras

39. Abadi M, Barham P, Chen J, Chen Z, Davis A, Dean J, et al. TensorFlow : A System for Large-Scale Machine Learning This paper is included in the Proceedings of the TensorFlow : A system for large-scale machine learning. Proc 12th USENIX Conf Oper Syst Des Implement. 2016; 272-283.

40. Glorot X, Statistics YBBT-P of the TIC on Al and. Understanding the difficulty of training deep feedforward neural networks. Teh YW, Titterington M, editors. PMLR ～; 2010. pp. 249-256. Available: http://proceedings.mlr.press/v9/glorot10a/glorot10a.pdf

41. Shi L, Reid LH, Jones WD, Shippy R, Warrington JA, Baker SC, et al. The MicroArray Quality Control (MAQC) project shows inter- and intraplatform reproducibility of gene expression measurements. Nat Biotechnol. 2006;24: 1151-1161. doi:10.1038/nbt1239

42. Tilgner H, Grubert F, Sharon D, Snyder MP. Defining a personal, allele-specific, and singlemolecule long-read transcriptome. Proc Natl Acad Sci. 2014;111: 9869-9874.

doi:10.1073/pnas.1400447111

43. Li H. Aligning sequence reads, clone sequences and assembly contigs with BWA-MEM. arXiv Prepr arXiv. 2013;00: 3. doi:10.1186/s13756-018-0352-y

44. Wu TD, Watanabe CK. GMAP: A genomic mapping and alignment program for mRNA and EST sequences. Bioinformatics. 2005;21: 1859-1875. doi:10.1093/bioinformatics/bti310

45. Hinrichs AS, Karolchik D, Baertsch R, Barber GP, Bejerano G, Clawson H, et al. The UCSC Genome Browser Database: update 2006. Nucleic Acids Res. 2006;34: D590-D598.

doi:10.1093/nar/gkj144

46. Kalkatawi M, Rangkuti F, Schramm M, Jankovic BR, Kamau A, Chowdhary R, et al. Dragon polya spotter: Predictor of poly(A) motifs within human genomic DNA sequences. Bioinformatics. 2012;28: 127-129. doi:10.1093/bioinformatics/btr602

47. Magana-Mora A, Kalkatawi M, Bajic VB. Omni-Polya: A method and tool for accurate recognition of poly(A) signals in human genomic DNA. BMC Genomics. 2017;18. doi:10.1186/s12864-0174033-7 
bioRxiv preprint doi: https://doi.org/10.1101/710699; this version posted April 23, 2020. The copyright holder for this preprint (which was not certified by peer review) is the author/funder, who has granted bioRxiv a license to display the preprint in perpetuity. It is made available under aCC-BY-NC-ND 4.0 International license.

\section{Supporting information}

648 S1 File. Supplementary material. This file contains all supplementary figures S1-10 Fig. 


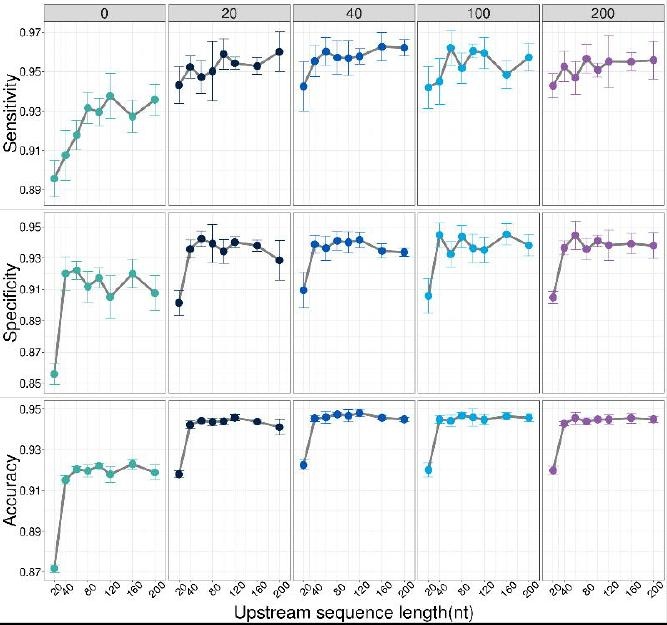




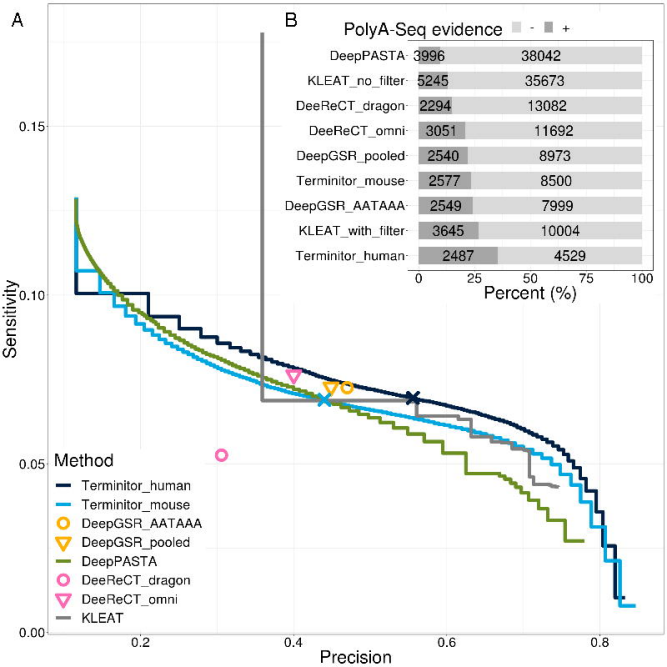




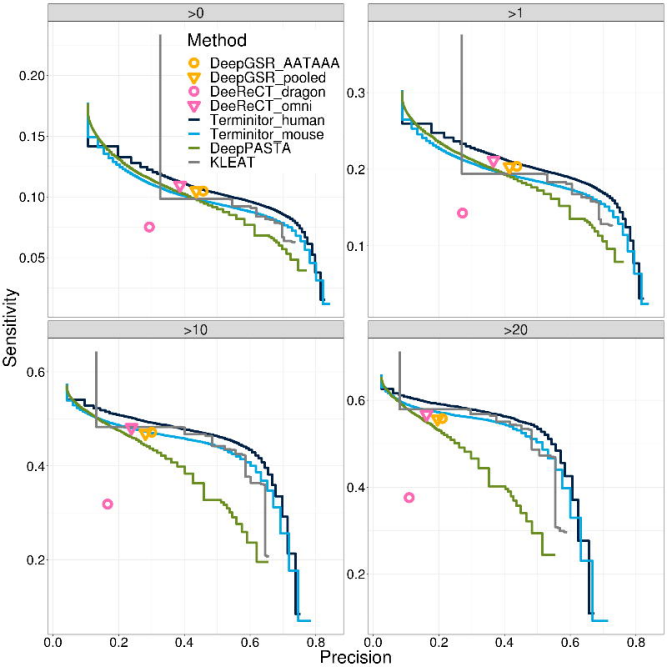


$>0$

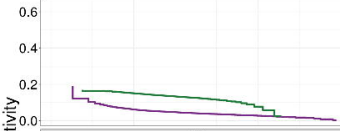

造 0.0

0.4

0.4
0.2
0.0

$0.4 \quad L$
0.2
0.0
0.00

$>10$
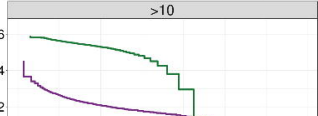

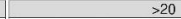

Sequencing

- Illumina

- PacBio

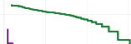

$\begin{array}{llll}0.25 & 0.50 & 0.75 & 0.00\end{array}$




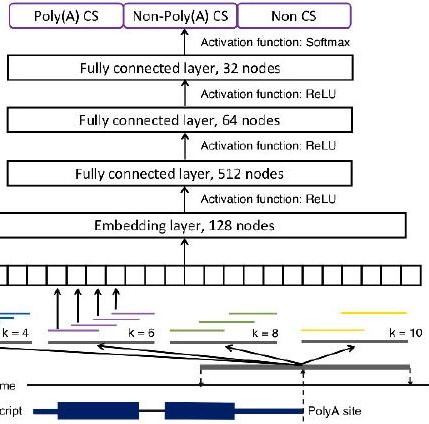

